\title{
A New High Resolution TVD Scheme for Unsteady Flows with Shock Waves ${ }^{1}$
}

R.A.B. QUEIROZ, 2 V.G. FERREIRA, ${ }^{3}$ R.G. CUENCA, 4 , Departamento de Matemática Aplicada e Estatística, Cx.P. 668, 13560-970 São Carlos, SP, Brasil.

\begin{abstract}
In this work, a new high resolution TVD scheme for unsteady flows with shock waves is presented. The performance of the scheme is investigated for solving Burgers and Euler's equations. In particular, 1D shock tubes, 1D inviscid turbulence (Burgers equation) and 2D supersonic/transonic flows are simulated. The numerical results show good agreement with numerical and experimental data.
\end{abstract}

Keywords. TVD, high-order upwind scheme, convective modelling.

\section{Introduction}

Approximation of convective transport is one unresolved and challenging issue in computational fluid dynamics (CFD) problems. According to Zijlema [18], the main problem is to reach (at the same time) accuracy, stability, monotonicity preservation, economy and algebraic simplicity. In this context, it is constructed a polynomial upwind scheme [12], called TOPUS (Third-Order Polynomial Upwind Scheme) for unsteady flows with shock waves. It generalizes the ISNAS (Interpolation Scheme which is Nonoscillatory for Advected Scalars) scheme of the Zijlema [18]. The construction of the TOPUS scheme is based on NVD (Normalized Variable Diagram) [10], and TVD (Total Variation Diminishing) constraints [7]. Consequently, it satisfies the CBC (Convection-Boundedness Criterion) [6]. The objective of this work is to present the mathematical formulation of the TOPUS and their applications in CFD problems. In particular, computations are presented for 1D Riemann problems for Euler and Burgers equations, and, as application, we simulated 2D supersonic/transonic flows. The organization of this work is as follows. In Section 2, the mathematical formulation of the TOPUS is described. A short outline of Burgers and Euler equations is presented in Section 3. In Section 4, 1D numerical experiments and their solutions are related. 2D aerodynamic flows are shown in Section 5. Section 6 contains our conclusions and discussions on future directions.

\section{The TOPUS Scheme}

For the construction of the TOPUS, we use the CBC constraints [6] and the recommendations of Leonard [10], that is the scheme passes at points $O(0,0)$,

\footnotetext{
${ }^{1}$ Supported by FAPESP: Grants 04/16064-9, 06/05910-1 and CNPq: Grant 304201/05-7.

${ }^{2}$ bonfim@icmc.usp.br, bonfimraf@gmail.com

${ }^{3}$ pvgf@icmc.usp.br

${ }^{4}$ rafaelgc@icmc.usp.br
} 
$Q(0.5,0.75), P(1,1)$ and has inclination of 0.75 at point $Q$. These four conditions plus a free condition were used for the determination of the TOPUS. In summary, the scheme in normalized variable (NV) of Leonard [10] is described as

$$
\hat{\phi}_{f}= \begin{cases}\alpha \hat{\phi}_{U}^{4}+(-2 \alpha+1) \hat{\phi}_{U}^{3}+\left(\frac{5 \alpha-10}{4}\right) \hat{\phi}_{U}^{2}+\left(\frac{-\alpha+10}{4}\right) \hat{\phi}_{U}, & \hat{\phi}_{U} \in[0,1], \\ \hat{\phi}_{U}, & \hat{\phi}_{U} \notin[0,1],\end{cases}
$$

where $\hat{\phi}_{U}$ is the upstream position in $\mathrm{NV}$, with respect to the $f$ face of the control volume (see details in references $[4,5]$ ). The adjustable constant $\alpha \in[-2,2]$ ensures that the TOPUS satisfies the CBC criterion. If $\alpha=2$, then TOPUS is entirely contained in the TVD region of Harten [7] and its corresponding flux limiter $\psi\left(r_{f}, \alpha\right)$ satisfies Sweby's monotonicity preservation condition when $r_{f}$ tends to 0 . If $\alpha=0$, then TOPUS corresponds to the ISNAS [18]. The flux limiter $\psi\left(r_{f}, \alpha\right)$ is deduced as follows. Let us consider the general approximation (first-order upwind plus an anti-diffusive term) to the numerical flux at the $f$ computational face

$$
\hat{\phi}_{f}=\hat{\phi}_{U}+\frac{1}{2} \psi\left(r_{f}, \alpha\right)\left(1-\hat{\phi}_{U}\right),
$$

where $r_{f}=\frac{\hat{\phi}_{U}}{1-\hat{\phi}_{U}}$ (a sensor). From equations (2.1) and (2.2),

$$
\psi\left(r_{f}, \alpha\right)=\frac{0.5\left(\left|r_{f}\right|+r_{f}\right)\left[(-0.5 \alpha+1) r_{f}^{2}+(\alpha+4) r_{f}+(-0.5 \alpha+3)\right]}{\left(1+\left|r_{f}\right|\right)^{3}} .
$$

This flux limiter has the advantage of being a smooth function of $r_{f}$ for $r_{f}>0$, so it may offer the best convergence behavior [18]. In case of $2 \mathrm{D}$ aerodynamic applications [3], $r_{f}=n u m^{ \pm} /$den and the flux limiter (2.3) can be rewritten by

$$
\Psi\left(\text { num }^{ \pm}, \operatorname{den}, \alpha, \epsilon_{L I M}\right)=\frac{0.5\left(\left|n u m^{ \pm}\right| \operatorname{den}+\text { num }^{ \pm}|\operatorname{den}|\right)\left(\Upsilon+\epsilon_{L I M}\right)}{\operatorname{den}\left(|\operatorname{den}|+\mid \text { num }^{ \pm} \mid\right)^{3}+\epsilon_{\text {LIM }}},
$$

where $\Upsilon=(-0.5 \alpha+1)\left(\text { num }^{ \pm}\right)^{2}+(\alpha+4)$ num $^{ \pm}$den $+(-0.5 \alpha+3)$ den $^{2}$ and $\epsilon_{L I M}$ is a limiter control (constant), allowing machine-zero steady-state convergence [3]. The new variables num $^{ \pm}$and den (the reader can see details in reference [3]) are den $=\left(q_{i}\right)_{k}-q_{i}$ and $n u m^{ \pm}=\hat{q}_{i}^{ \pm}-q_{i}$, where $q$ is a generic property.

\section{Mathematical Modelling}

1D shock tube: This problem is modeled by

$$
\mathbf{U}_{t}+\mathbf{F}_{x}=0
$$

where $\mathbf{U}=(\rho, \rho u, e)^{T}, \mathbf{F}=\left(\rho u, \rho u^{2}+p, u(e+p)\right)^{T}$ and $p=(0.4)\left(e-\frac{1}{2} \rho u^{2}\right)$. The variables $\rho, u, e, p$ are, respectively, the density, velocity, total energy and pressure. 
1D inviscid turbulence: This problem is formulated by

$$
u_{t}+\left(\frac{u^{2}}{2}\right)_{x}=0
$$

with initial condition $u(x, 0)=u_{L}$ for $x<0$ and $u(x, 0)=u_{R}$ for $x>0$ (see reference [1]). In the view of the entropy condition the exact solution is either a shock wave, when $u_{L}>u_{R}$, or a rarefaction wave, when $u_{L} \leq u_{R}$. In case the shock wave, the solution is given by [1]

$$
u(x, t)= \begin{cases}u_{L}, & \text { if } x-0.5\left(u_{L}+u_{R}\right) t<0 \\ u_{R}, & \text { if } x-0.5\left(u_{L}+u_{R}\right) t>0\end{cases}
$$

In case rarefaction wave, the solution is given by [1]

$$
u(x, t)= \begin{cases}u_{L}, & \text { if } \frac{x}{t} \leq u_{L}, \\ \frac{x}{t}, & \text { if } u_{L}<\frac{x}{t}<u_{R}, \\ u_{R}, & \text { if } \frac{x}{t} \geq u_{R} .\end{cases}
$$

2D transonic and supersonic flows: This problem is given by

$$
\mathbf{Q}_{t}+\mathbf{E}_{x}+\mathbf{F}_{y}=0
$$

where $\mathbf{Q}=[\rho, \rho u, \rho v, e]^{T}, \mathbf{E}=\left[\rho u, \rho u^{2}+p, \rho u v,(e+p) u\right]^{T}, e_{i}=\frac{p}{(\gamma-1) \rho}, e=\rho\left(e_{i}+\right.$ $\left.0.5\left(u^{2}+v^{2}\right)\right)$ and $\mathbf{F}=\left[\rho v, \rho u v, \rho v^{2}+p,(e+p) v\right]^{T}$. The details of the numerical methods for solver equations (3.1) and (3.3) are presented in references [8]. The solver for equation (3.2) is constructed using an explicit finite difference method, being TOPUS used for the non-linear convective term.

\section{1D Numerical Experiments}

\subsection{Shock Tubes}

Sod's shock tube [14]: The solution of this problem consists of a left rarefaction, a contact and a right shock. In the numerical simulations, it is considered equation (3.1) with initial condition given by

$$
(\rho, u, p)^{T}= \begin{cases}(1,0,1)^{T}, & 0 \leq x<0.5 \\ (0.125,0,0.1)^{T}, & 0.5 \leq x \leq 1\end{cases}
$$

We used in the simulation a mesh size of $N=800$ computational cells, Courant number (denoted by $\theta$ ) 0.6 and final time $t=0.2$. The numerical results and reference solution (obtained by first order upwind scheme with $N=2000$ ) are presented in Figure 1. One observes from this figure that the numerical results obtained with the TOPUS limiter $\psi\left(r_{f}, 2\right)$ are in good agreement with reference solution, without oscillations. 


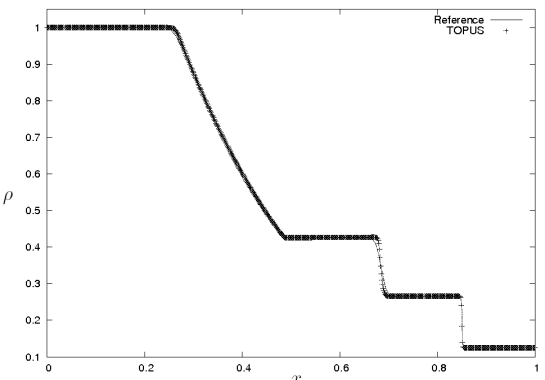

(a) Density distribution $\rho$.

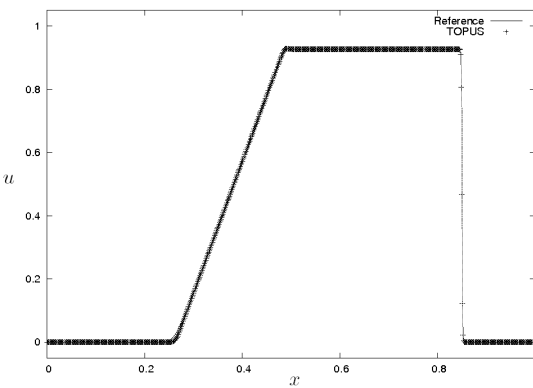

(b) Velocity $u$.

Figure 1: Numerical results and reference solution for Sod's shock tube.

Shu-Osher's shock tube [13]: This problem describes a shock interacting with a sinusoidal density field. It is considered equation (3.1), $x \in[-1,3]$, with the following initial condition

$$
(\rho, u, p)^{T}=\left\{\begin{array}{lr}
(3.86,2.63,10.33)^{T}, & -1 \leq x<-0.8 \\
(1+0.2 \sin (5 x), 0,1)^{T}, & -0.8 \leq x \leq 3 .
\end{array}\right.
$$

This test case provides a good one for examinating the performance of a high order upwind scheme, because it possesses several extrema in the smooth regions. In the simulation, it was used a uniform mesh of $N=250$ cells, Courant number $\theta=0.6$ and time $t=1.0$. The solutions are presented in Figure 2. Once again, the results using TOPUS limiter $\psi\left(r_{f}, 2\right)$ is a good concordance with the reference solution.

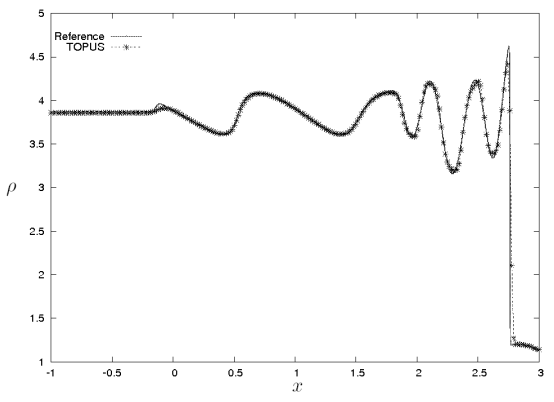

(a) Density distribution $\rho$.

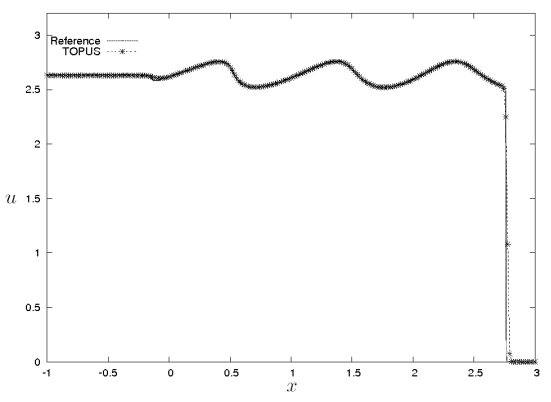

(b) Velocity $u$.

Figure 2: Numerical results and reference solution for Shu-Osher's shock tube.

Toro's shock tube [15] - test case 1: This test is actually the left half of the blast wave problem of Woodward and Colella [17]. It has solution that consists of a strong shock wave, a contact surface and a left rarefaction wave. In the 
numerical process, it is considered equation (3.1) with the initial condition given by

$$
(\rho, u, p)^{T}=\left\{\begin{array}{lc}
(1,0,1000)^{T}, & 0 \leq x<0.5 \\
(1,0,0.01)^{T}, & 0.5 \leq x \leq 1 .
\end{array}\right.
$$

Figure 3 depicts the reference solution and the numerical results for density produced by TOPUS limiter $\psi\left(r_{f}, 2\right)$. In the simulation, it was used a uniform mesh of 800 computational cells, two Courant numbers $(\theta=0.2$ and $\theta=0.6)$ and time $t=0.012$. One can see from this figure that the TOPUS provides good results for both values of Courant number.

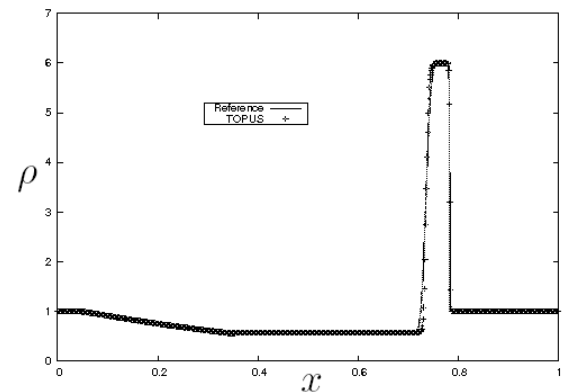

(a) $\theta=0.2$

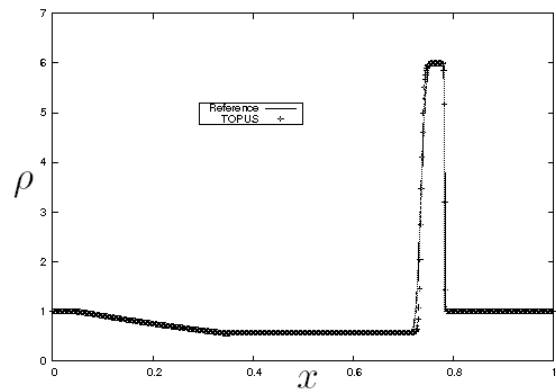

(b) $\theta=0.6$.

Figure 3: Numerical results and reference solution for density distribution.

Toro's shock tube problem [15] - test case 2: This last shock tube investigated is formulated by equation (3.1) with $x \in[0,1]$ and initial condition

$$
(\rho, u, p)^{T}= \begin{cases}(5.99924,19.5975,460.894)^{T}, & 0 \leq x<0.4 \\ (5.99242,-6.19633,46.0950)^{T}, & 0.4 \leq x \leq 1\end{cases}
$$

The solution of this shock tube consists of three strong discontinuities traveling to the right. Figure 4 shows the numerical results for density obtained with the TOPUS limiter $\psi\left(r_{f}, 2\right)$. The uniform mesh used for this problem was the 800 computational cells, time $t=0.035$ and Courant numbers $\theta=0.2$ and $\theta=0.6$. Once again, for this problem, it can be seen from this figure that the TOPUS provides a satisfactory solution.

\subsection{Inviscid Turbulence}

Test case 1: In the numerical process, it is considered the equation (3.2) with the initial condition given by $u_{L}=0.8$ and $u_{R}=0.2$. It was adopted for solving this problem a mesh size of 100 computational cells, $d x=0.02, d t=0.018$, time $t=0.5, x \in[-1,1]$ and TOPUS limiter $\psi\left(r_{f}, 2\right)$ for non-linear terms. The numerical result and the exact solution are presented in Figure 5-(a). This figure shows a good concordance between the numerical solution and the exact solution. Figure 


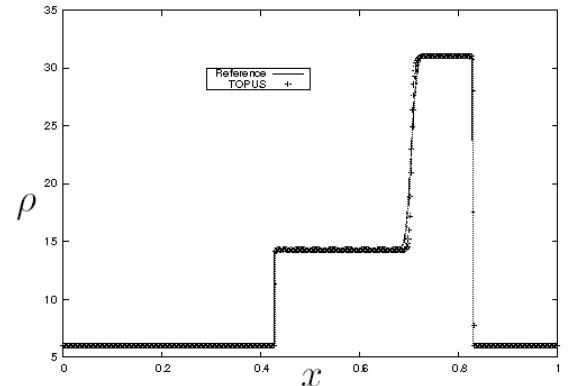

(a) $\theta=0.2$

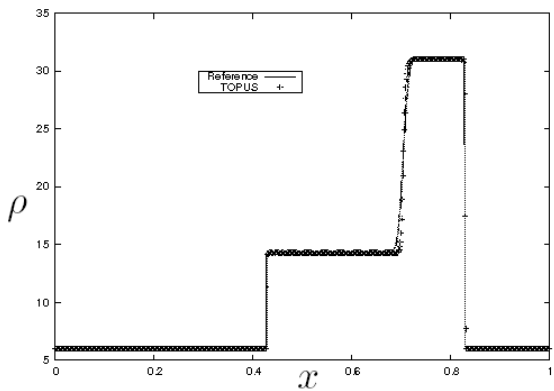

(b) $\theta=0.6$

Figure 4: Numerical results and reference solution for density distribution.

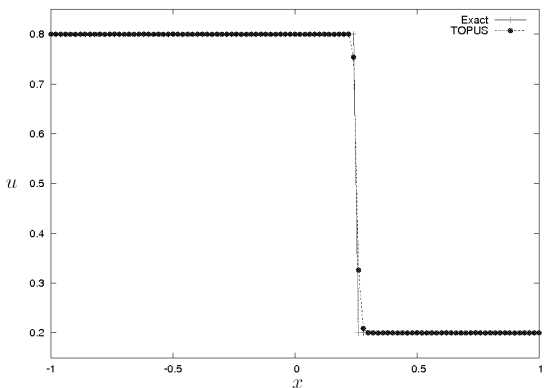

(a) Time $t=0.5$.

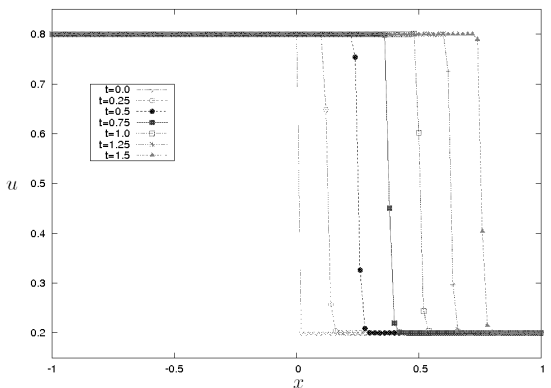

(b) Transient profile.

Figure 5: Solutions for equation (3.2) using various simulation time.

5-(b) shows the transient profile. One can conclude from this figure that the numerical method using TOPUS is stable and does not introduce spurious oscillations.

Test case 2: In the numerical simulation, it is solved equation (3.2) with the initial condition given by $u_{L}=0.0$ and $u_{R}=0.5$. It was adopted TOPUS limiter $\psi\left(r_{f}, 2\right)$, a mesh with $N=200$ computational cells, final time $t=2, x \in[-1.5,1]$, Courant numbers $\theta=0.3$ and $\theta=0.9$. The numerical result and the exact solution are presented in Figure 6. As in the test case 1, this figure shows good accuracy of the numerical solution.

\section{2D Aerodynamic Flows}

\subsection{Diamond supersonic airfoil}

The well-known theoretical result for supersonic aerodynamic flow is the oblique shock wave in diamond airfoil [2]. In the present study, the oblique shock is simulated with wall deflection angle of $10^{\circ}$ degrees, free-stream Mach $=1.5$ and TOPUS 


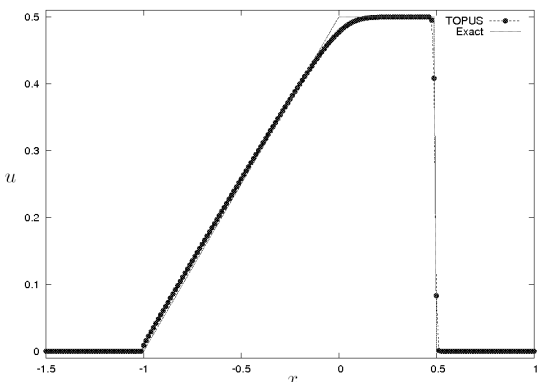

(a) $\theta=0.3$.

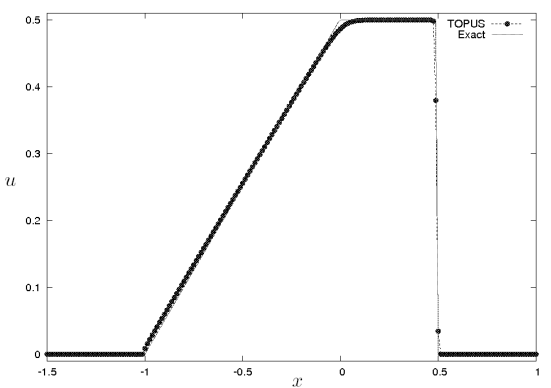

(b) $\theta=0.9$

Figure 6: Test case 2: numerical and exact solutions using two Courant numbers.

limiter $\Psi\left(n u m^{ \pm}, \operatorname{den}, 2,10^{-8}\right)$ for approximating non-linear terms. The theoretical result [2] gives a shock wave angle of $56.5^{\circ}$ degree. One can see from Table 1 that the numerical results using the van Albada [16] and TOPUS limiters are consistent with theoretical one, using both conserved (Cons) and primitive (Prim) variable reconstructions. Figure 7 depicts the convergence history of the numerical method.

Table 1: 2D supersonic flow: numerical results of the oblique shock wave.

\begin{tabular}{|c||c|}
\hline Flux limiter & Oblique shock wave \\
\hline van Albada - Cons & $56.74^{\circ}$ \\
van Albada - Prim & $56.79^{\circ}$ \\
TOPUS - Cons & $57.53^{\circ}$ \\
TOPUS - Prim & $57.04^{\circ}$ \\
\hline
\end{tabular}

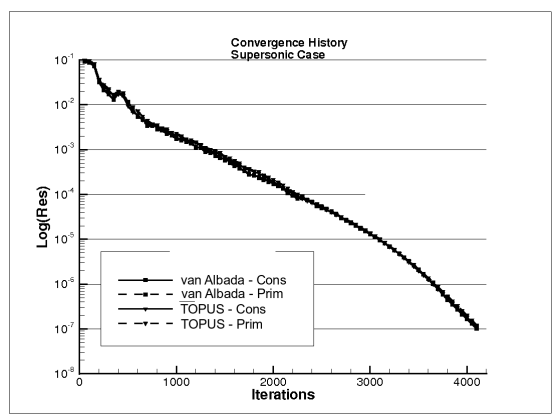

Figure 7: Convergence history supersonic simulations. 


\subsection{RAE2822 transonic airfoil}

In the present application, a "O" type structured mesh [9] on RAE2822 airfoil, at Mach $0.725,2.310^{\circ}$ angle of attack and TOPUS limiter $\Psi\left(\right.$ num $^{ \pm}$, den $\left., 2,10^{-8}\right)$ were adopted [11]. The convergence history in this case, using TOPUS and van Albada limiters, is presented in Figure 8-(a). It is clear from this figure that, in the two reconstructions, the convergence was not affected by flux limiters. Figure 8-(b) shows the comparison between the numerical results with experimental data of [11], for the pressure coefficient $\left(C_{p}\right)$. One can note from this figure that TOPUS and van Albada limiters provided similar results for two reconstructions. These results are also in good agreement with the experimental data of [11]. Furthermore, using these limiters and the two reconstructions, Figure 9 presents the shock detail, calculated for Mach number (MACH) and pressure $(\mathbf{P})$ distributions around airfoil. It is seen that both methods produce good results, showing little disagreement at the shock region.

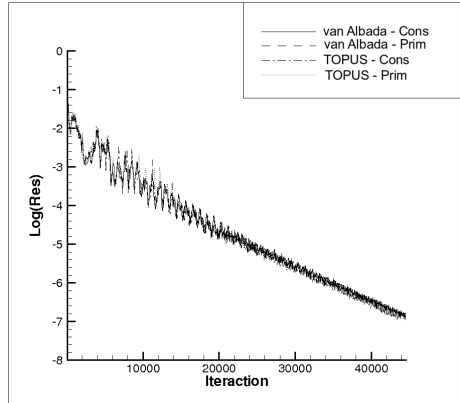

(a) Convergence history.

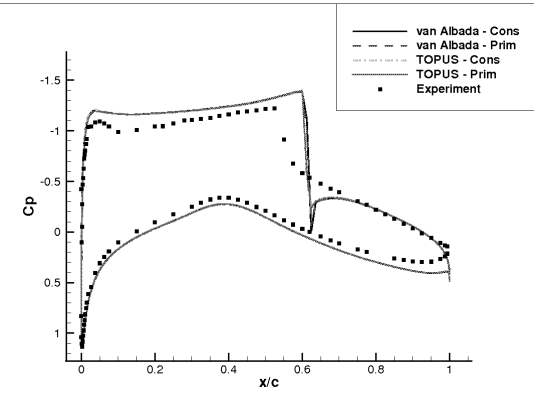

(b) Pressure coefficient.

Figure 8: Numerical and experimental results for 2D transonic aerodynamic flow.

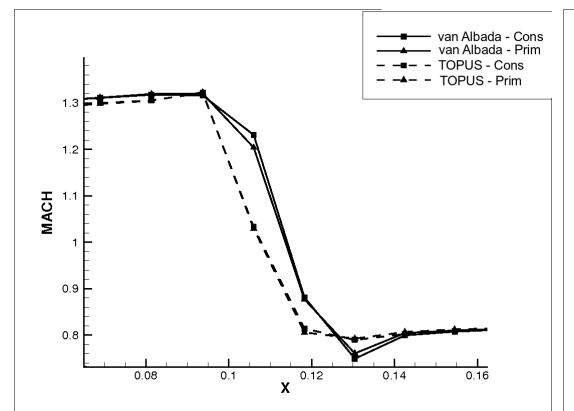

(a) Mach number distribution.

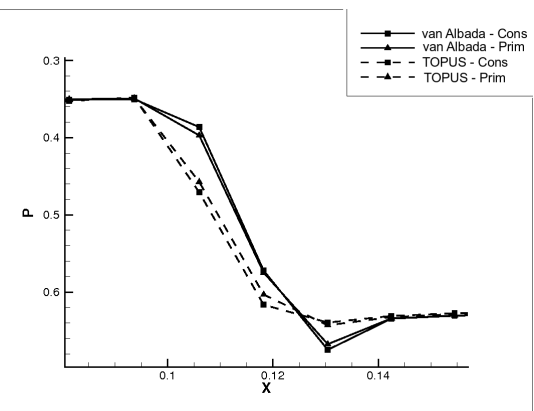

(b) Pressure distribution.

Figure 9: Shock detail around a RAE2822 transonic airfoil. 


\section{Conclusion}

In this work, we have presented a new high resolution TVD scheme for unsteady flows with shock waves. The scheme was tested in 1D/2D Euler and 1D Burgers equations. From 1D numerical results, one can conclude that the TOPUS is a robust strategy to capture shock, when compared with the reference solution. For supersonic and transonic flows, the TOPUS provided result compatible with that the van Albada limiter and experimental data. For the future, we are planning to apply TOPUS scheme for solution of turbulent and viscoelastic free surface flows.

Resumo. O trabalho apresenta um novo esquema polinomial upwind TVD para simular escoamentos transientes com choques. O desempenho do esquema é investigado em problemas 1D/2D modelados pelas equações de Euler e Burgers. Os resultados obtidos mostram que o TOPUS é adequado para resolver essas EDPs.

\section{References}

[1] R. Ahmed, "Numerical Schemes Applied to the Burgers and Buckley-Leverett Equations", Msc dissertation, University of Reading, 2004.

[2] J.D. Anderson, "Modern Compressible Flow: With Historical Perspective", McGraw-Hill, 1990.

[3] E.D.V. Bigarella, "Advanced Turbulence Modeling for Complex Aerospace Applications", Ph.D. Thesis, Instituto Tecnológico de Aeronáutica, São José dos Campos, SP, 2007.

[4] V.G. Ferreira, F.A. Kurokawa, R.A.B. Queiroz, M.K. Kaibara, C.M. Oishi, J.A. Cuminato, A. Castelo, M.F. Tomé, S. McKee, Assessment of a high-order finite difference upwind scheme for the simulation of convection-diffusion problems, Int. J. Numer. Meth. Fluids, In Press.

[5] V.G. Ferreira, C.M. Oishi, F.A. Kurokawa, M.K. Kaibara, J.A. Cuminato, A. Castelo, N. Mangiavacchi, M.F. Tomé, S. McKee, A combination of implicit and adaptative upwind tools for the numerical solution of incompressible free surface flows, Comm. Numer. Meth. Eng., 23 (2007), 419-445.

[6] P.H. Gaskell, A.K. Lau, Curvature-compensated convective transport: SMART, a new boundedness-preserving transport algorithm, Int. J. Numer. Meth. Fluids, 8 (1988), 617-641.

[7] A. Harten, High resolution schemes for conservation laws, J. Comput. Phys, 49 (1983), 357-393.

[8] C. Hirsch, "Numerical Computation of Internal and External Flows", Fundamentals of Numerical Discretization, John Wiley, Vol.1 and Vol.2, 1991.

[9] A. Jameson, W. Schimidt, E. Turkel, Numerical solutions of the Euler equations by finite volume methods using Runge-Kutta time stepping schemes, AIAA J., 81 (1981), 1253-1259. 
[10] B.P. Leonard, Simple high-accuracy resolution program for convective modeling of discontinuities, Int. J. Numer. Meth. Fluids, 8 (1988), 1291-1318.

[11] NPARC Alliance Validation Archive, http://www.grc.nasa.gov/WWW/wind/valid/raetaf/raetaf04/raetaf04.html. Access: $03 / 31 / 2008$.

[12] R.A.B. Queiroz, F.A. Kurokawa, V.G. Ferreira, F.P. Martins, Development and implementation of polynomial scheme for the numerical solution of 1D conservation laws, in "Proceedings of the VIII Simpósio Mecânica Computacional", SIMMEC, Belo Horizonte, 2008.

[13] C.W. Shu, S. Osher, Efficient implementation of essentially non-oscillatory shock capturing schemes, J. Comput. Phys, 83 (1989), 32-78.

[14] G. Sod, A survey of several finite difference methods for systems of nonlinear hyperbolic conservation laws, J. Comput. Phys, 27 (1978), 1-31.

[15] E.F. Toro, "Riemann Solves and Numerical Methods for Fluid Dynamics", 2nd Edition, Springer-Verlag, Berlin, 1999.

[16] G.D. van Albada, B. van Leer, W.W. Roberts, A comparative study of computational methods in cosmic gas dynamics, Astron. Astrophys., 108 (1982), 76-84.

[17] P. Woodward, P. Colella, The numerical simulation of two-dimensional fluid flow with strong shocks, J. Comput. Phys, 24 (1984), 115-173.

[18] M. Zijlema, On the construction of a third-order accurate monotone convection scheme with application to turbulent flows in general domains, Int. J. Numer. Meth. Fluids, 22 (1996), 619-641. 